\title{
Optimal Time-point of Response Assessment for Predicting Survival Is Associated With Tumor Burden in Hepatocellular Carcinoma Receiving Repeated Transarterial Chemoembolization
}

\section{Dongdong Xia}

Xi'an International Medical Center

\section{Qiuhe Wang}

Xijing Hospital Department of Liver Diseases and Digestive Interventional Radiology

\section{Wei Bai}

Xi'an International Medical Center

\section{Enxin Wang}

Fourth Military Medical University: Air Force Medical University

\section{Zhexuan Wang}

Xijing Hospital

\section{Wei Mu}

Third Military Medical University Southwest Hospital

Junhui Sun

Zhejiang University School of Medicine First Affiliated Hospital

Ming Huang

Tumor Hospital of Yunnan Province: Yunnan Cancer Hospital

\section{Guowen Yin}

Jiangsu Cancer Institute and Hospital: Jiangsu Cancer Hospital

\section{Hailiang Li}

Henan Provincial Tumor Hospital: Henan Cancer Hospital

Hui Zhao

Nantong University Affiliated Hospital: Affiliated Hospital of Nantong University

\section{Chunqing Zhang}

Shandong Provincial Hospital

\section{Jing Li}

Third Military Medical University Second Affiliated Hospital: Xinqiao Hospital

\section{Jianbing Wu}

Nanchang University Second Affiliated Hospital

\section{Xiaoli Zhu}

Soochow University Affiliated No 1 Peoplel's Hospital: First Affiliated Hospital of Soochow University

\section{Shufa Yang}

Tumor Hospital of Xinjiang Autonomous Region: Xinjiang Medical University Affiliated Tumor Hospital

\section{Xingnan Pan}

180th Hospital of PLA 
Jiaping Li

Sun Yat-sen University First Affiliated Hospital

\section{Zixiang Li}

Affiliated Hospital of Medical College Qingdao University: The Affiliated Hospital of Qingdao University

\section{Guohui Xu}

Sichuan Cancer Hospital and Research Institute: Sichuan Cancer Hospital and Institute

\section{Haibin Shi}

Jiangsu Province Hospital and Nanjing Medical University First Affiliated Hospital

\section{Hui Zhanf}

Third Military Medical University Southwest Hospital

\section{Yuelin Zhanf}

Zhejiang University School of Medicine First Affiliated Hospital

\section{Rong Ding}

Tumor Hospital of Yunnan Province: Yunnan Cancer Hospital

\section{Hui Yu}

Jiangsu Cancer Institute and Hospital: Jiangsu Cancer Hospital

\section{Lin Zheng}

Henan Provincial Tumor Hospital: Henan Cancer Hospital

\section{Xiaohu Yang}

Nantong University Affiliated Hospital: Affiliated Hospital of Nantong University

\section{Guangchuan Wang}

Shandong Provincial Hospital

\section{Nan You}

Third Military Medical University Second Affiliated Hospital: Xinqiao Hospital

\section{Long Feng}

Nanchang University Second Affiliated Hospital

\section{Shuai Zhang}

Soochow University Affiliated No 1 Peoplel's Hospital: First Affiliated Hospital of Soochow University

\section{Wukui Huang}

Tumor Hospital of Xinjiang Autonomous Region: Xinjiang Medical University Affiliated Tumor Hospital

\section{Tao Xu}

180th hospital of PLA

\section{Wenzhe Fan}

Sun Yat-sen University First Affiliated Hospital

\section{Xueda Li}

The Affiliated Hospital of Qingdao University

\section{Xuegang Yang}

Sichuan Cancer Hospital and Research Institute: Sichuan Cancer Hospital and Institute

\section{Weizhong Zhou}

Jiangsu Province Hospital and Nanjing Medical University First Affiliated Hospital

\section{Xiaomei Li}

Xi'an International Medical Center

\section{Bohan Luo}

Xi'an International Medical Center 


\section{Yong LV}

Xijing Hospital Department of Liver Diseases and Digestive Interventional Radiology

\section{Kai Li}

Xijing Hospital Department of Liver Diseases and Digestive Interventional Radiology

\section{Wengang Guo}

Xi'an International Medical Center

\section{Zhanxin Yin}

Xi'an International Medical Center

\section{Daiming Fan}

Fourth Military Medical University: Air Force Medical University

\section{Jielai Xia}

Fourth Military Medical University: Air Force Medical University

\section{Guohong Han ( $\sim 13991969930 @ 126 . c o m$ )}

Xi'an International Medical Center

\section{Research Article}

Keywords: TACE, objective response, tumor burden, HCC, surrogate endpoints

Posted Date: July 7th, 2021

DOI: https://doi.org/10.21203/rs.3.rs-671117/v1

License: (c) (i) This work is licensed under a Creative Commons Attribution 4.0 International License. Read Full License

Version of Record: A version of this preprint was published at European Radiology on April 6th, 2022. See the published version at https://doi.org/10.1007/s00330-022-08716-4. 


\section{Abstract}

Background

Objective response rate (ORR) under mRECIST criteria after transarterial chemoembolization (TACE) has been identified as a surrogate endpoint of overall survival (OS). However, its optimal time-point remains controversial and may be influenced by tumor burden. We aim to investigate the surrogacy of initial/best ORR in relation to tumor burden.

Methods

A total of 1549 eligible treatment-naïve patients with unresectable hepatocellular carcinoma (HCC), Child-Pugh score $\leq 7$, and performance status score $\leq 1$ undergoing TACE between January 2010 and May 2016 from 17 academic hospitals were retrospectively analyzed.

Results

Both initial and best ORRs interacted with tumor burden defined as our previously proposed "six-and-twelve" criteria. Both initial and best ORRs could equivalently predict and correlated with OS in low (adjusted HR: 2.55 and 2.95, respectively, both $\mathrm{P}<0.001 ; \mathrm{R}=0.84, \mathrm{P}=0.035$ and $\mathrm{R}=0.97, \mathrm{P}=0.002$, respectively) and intermediate tumor burden strata (adjusted HR: 1.81 and 2.22, respectively, both $P<0.001 ; R=0.74, P=0.023$, and $R=0.9, P=0.002$, respectively). For high strata, only best ORR exhibited qualified prognostic value (adjusted HR: 2.61, $\mathrm{P}<0.001$ ) with a satisfying correlation $(R=0.70, P=0.035)$, whereas initial ORR was not statistically significant (adjusted HR: $1.08 P=0.357 ; R=0.22, P=0.54$ ).

Conclusions

ORR after TACE as a surrogate of OS is closely associated with tumor burden. For patients with low/intermediate tumor burden, initial ORR should be preferred over best ORR in its early availability upon similar sensitivity; whereas for patients with high tumor burden, best ORR has optimal sensitivity. Timing of OR assessment should be tailored according to tumor burden in future clinical trials and practice.

\section{Introduction}

Transarterial chemoembolization (TACE) is the recommended first-line non-curative therapy for unresectable hepatocellular carcinoma (HCC), and overall survival (OS) is the standard outcome measurement(1, 2). However, setting OS as an exclusive endpoint would increase the difficulty of clinical researches since it requires a large sample size and long follow-up duration to capture sufficient events(3). Consequently, with early availability and close correlation to survival $(4,5)$, objective response (OR) has been proposed as a robust surrogate endpoint $(1,6)$, which is defined by the sum of complete and partial response (CR and PR) under modified Response Evaluation Criteria in Solid Tumours (mRECIST) criteria(7-10).

Nevertheless, the common practice of repeated "on-demand" TACE arouses a question on whether the surrogacy of OR should depend on response after the initial procedure (initial OR) or the best response achieved during repeated procedures (best OR)(11). Currently, initial and best responses are sometimes bracketed together to predict survival(4, $10,12,13)$. However, inconsistent timing of response assessment across studies lead to variable OR rates (ORRs) and compromise its surrogacy, calling for determination of the optimal timing. Theoretically, initial response could exert surrogacy at the earliest time available, and has been supported by a recent study(14). However, in this study, subsequent responders without initial OR still exhibited better outcome than persistent non-responders, indicating 
initial response may leave out survival benefits achieved in subsequent responders $(9,14,15)$. On the other hand, several studies favoring best $\operatorname{ORR}(14,16)$ reveals controversies on this issue (Supplementary table 1)(11).

An underlying cause of these controversies is the influence of tumor burden on TACE schedules. OR after initial TACE is more likely applied for small solitary nodule, obviating the necessity for subsequent procedure and evaluation; whereas large or multiple nodules usually require repeated TACE due to incomplete necrosis or viable tumors after initial procedure, and best ORR becomes more intuitively accurate(17). Hence it is reasonable to hypothesize that tumor burden, a particularly heterogeneous characteristic for TACE candidates, may influence the optimal timing of ORR assessment to predict survival(11).

The current study aims to investigate the prognostic value of initial and best ORR and their surrogacy in relation to tumor burden using individual data.

\section{Methods}

\section{Patient eligibility}

Between January 2010 and May 2016, 3794 consecutive patients with unresectable HCC undergoing TACE from 17 Chinese tertiary centers were screened. HCC was diagnosed per AASLD and EASL guidelines $(1,2)$. Inclusion criteria were: (1) treatment-naïve; (2) at least one nodule > $1 \mathrm{~cm}$; (3) Eastern Cooperative Oncology Group performance status (ECOG PS) score 0-1; and (4) Child-Pugh score A5-B7. Exclusion criteria were: (1) vascular invasion or extrahepatic spread; (2) spontaneous tumor rupture; (3) other malignancies; (4) combined systematic or loco-regional therapy; (5) absence of baseline or follow-up imaging; and (6) decompensated portal hypertension. The diameter of the largest tumor (tumor size, Ts) and tumor number (Tn) were measured by two independent central reviewers (WB and DX) using dynamic contrast-enhanced computed tomography (CT) or magnetic resonance (MR) images, both of whom were blinded to patients' clinical data. Tumor burden was defined as Ts + Tn, in accordance with our previous study, namely "six-and twelve" score, the sum of tumor size and number $\leq 6$; the sum > 6 but $\leq 12$; the sum $>12$ for low/intermediate/high tumor burden, respectively(18).

\section{Treatment procedure}

TACE procedures, were performed by investigators with at least 8 years of experience. Specifically, chemotherapeutic drugs used to mix with lipiodol (3-30ml) included doxorubicin (10-50 mg), cisplatin (10-110 mg), epirubicin (10-50 $\mathrm{mg}$ ), or oxaliplatin (100-200 mg), and were selected according to the practice of each center. Gelatin sponge or polyvinyl alcohol foam particles were introduced for embolization, which was preferably super-selective. When necessary in treating large or multiple tumors, split TACE was allowed within 4-6 weeks of the first TACE session, and sequential TACE would be conducted with the same agents upon following-up identification of intrahepatic viable tumors or intrahepatic recurrences by CT/MRI according to "on demand" schedule, if patients' performance status and laboratory findings permitted. TACE failure/refractory was defined as the appearance of vascular invasion or extrahepatic spread, deterioration of liver function with Child-Pugh grade C,consistent progressive disease (PD) after three sessions of TACE within 6 months $(19,20)$. Following treatment would be subsequently recommended for patients with TACE failure/refractory, and administered upon patients' choice and consent.

\section{Evaluation and definitions of the treatment response}

Radiological response was routinely assessed at 4-6 weeks after first TACE procedure using dynamic contrastenhanced CT or MRI to obtain initial response, and every 4-12 weeks thereafter (depending on each center's practice, patient characteristics and response) for subsequent assessment using consistent imaging modality. Initial response 
was defined as the radiological response after the first session of TACE, regardless of the schedule of cycles(7, 8, 14, $21,22)$. Best response was defined as the best radiological result across all time points during 'on-demand' repeated sessions(14-16). In cases where the same response was maintained through sessions of the procedure without improvement, the initial response would be regarded as the best response. All data acquisition of response assessment at individual patient, including initial and best response, was evaluated by two independent central reviewers (WB and DX) blinded to other clinical data to avoid bias, and final consensus with another reviewer (EW) would be reached when the two reviewers had different judgements. Complete response (CR), partial response (PR), stable disease (SD) and progressive disease (PD) were defined by mRECIST criteria $(6,23)$. For analysis, the overall response (combined responses of target and non-target lesions) was adopted in our study. OR referred to sum of CR and PR, whereas non-response referred to sum of SD and PD. Patients also were divided into four groups according to occurrence and time of objective response as follows; (1) patients who initially achieved CR after the first TACE (referred to "initial CR"); (2) patients who subsequently achieved CR after at least two sessions (referred to "subsequent CR"); (3) patients who achieved PR after multiple sessions of TACE (referred to "subsequent PR") and (4) patients who did not achieve objective response during the treatment course (referred to "persistent non-response'.

\section{Statistics}

Missing values were imputed with 5 independent draws. Quantitative variables were compared with Student's t-test or Mann-Whitney U test, whereas categorical variables were compared by chi-squared test or Fisher's exact test. OS, defined as the time interval between the initial TACE and all-cause death, was calculated with Kaplan-Meier method and compared by log-rank test. Patients who survived at last follow-up date (December 15th, 2017) or lost to follow-up were censored. Cox proportional regression was performed to identify the prognostic factors and hazard ratio (HR) with $95 \%$ confidence interval $(95 \% \mathrm{Cl})$. Notably, in Cox regression analyses. Evolutionary events were addressed as time-dependent covariates. Multivariate binary logistic regression was used to identify predictors of non-response after initial TACE. Interaction between significant predictors and initial/best ORR were tested. The relationship between the survival probability (in deciles) and odds of initial/best ORR ([p/1-p], where $p$ is initial/best ORR) was evaluated using Pearson's correlation coefficient R and linear regression. Since ORR of $100 \%$ would result in odds values of positive infinity, corresponding survival decile groups would be integrated into the adjacent group until the integrated ORR became less than $100 \%$, and overall odds of this integrated group was calculated accordingly(24), resulting in less than 10 decile groups in some analyses. Significance level was two-sided $P<0.05$. Statistical analyses were conducted using SPSS version 22 (IBM, Somers, New York, USA) and R version 3.2.5.

\section{Results}

\section{Patient characteristics}

A total of 1549 patients were finally included (Supplementary Fig. 1). As is shown in Table 1, the median age was 57 (interquartile range, IQR: 48-65) years, and 1047 (67.6\%) patients presented ECOG PS 0. The median TS was 6.1 (IQR: 3.9-9.6) cm, and 895 (57.8\%) had a single nodule. Approximately 95\% patients were graded Child-Pugh A.

Percentages and patterns of missing values are shown in Supplementary Fig. 2. The median sessions of TACE was 3 (IQR: 2-4), and the median follow-up time was 17.3 (IQR: 7.6-30.5) months. 
Table 1

Baseline demographics and clinical characteristics in 1549 patients

\begin{tabular}{|c|c|c|c|c|}
\hline Variables & Total $(N=1549)$ & $\begin{array}{l}\text { Patients with low } \\
\text { tumor burden }(\mathrm{N}= \\
\text { 433) }\end{array}$ & $\begin{array}{l}\text { Patients with } \\
\text { intermediate tumor } \\
\text { burden }(\mathrm{N}=755)\end{array}$ & $\begin{array}{l}\text { Patients with high } \\
\text { tumor burden }(\mathrm{N}= \\
\text { 361) }\end{array}$ \\
\hline Age, years old & $57(48-65)$ & $57(49-65)$ & $58(48-66)$ & $55(45-62)$ \\
\hline $\begin{array}{l}\text { Gender } \\
\text { (male/female) }\end{array}$ & $\begin{array}{l}1314 / 235 \\
(84.8 \% / 15.2 \%)\end{array}$ & $\begin{array}{l}345 / 88 \\
(79.7 \% / 20.3 \%)\end{array}$ & $\begin{array}{l}656 / 99 \\
(86.9 \% / 13.1 \%)\end{array}$ & $\begin{array}{l}313 / 48 \\
(86.7 \% / 13.3 \%)\end{array}$ \\
\hline $\begin{array}{l}\text { Aetiology } \\
\text { (HBV/others) }\end{array}$ & $\begin{array}{l}1380 / 169 \\
(89.1 \% / 10.9 \%)\end{array}$ & $\begin{array}{l}398 / 35 \\
(91.9 \% / 8.1 \%)\end{array}$ & $\begin{array}{l}668 / 87 \\
(88.5 \% / 11.5 \%)\end{array}$ & $\begin{array}{l}314 / 47 \\
(87 \% / 13 \%)\end{array}$ \\
\hline $\begin{array}{l}\text { ECOG PS score } \\
(0 / 1)\end{array}$ & $\begin{array}{l}1047 / 502 \\
(67.6 \% / 32.4 \%)\end{array}$ & $\begin{array}{l}336 / 97 \\
(77.6 / 22.4 \%)\end{array}$ & $\begin{array}{l}523 / 232 \\
(69.3 \% / 30.7 \%)\end{array}$ & $\begin{array}{l}188 / 173 \\
(52.1 \% / 47.9 \%)\end{array}$ \\
\hline $\begin{array}{l}\text { Child-Pugh score } \\
(5 / 6 / 7)\end{array}$ & $\begin{array}{l}1186 / 287 / 76 \\
(76.6 \% / 17.9 \% / 4.9 \%)\end{array}$ & $\begin{array}{l}322 / 79 / 28 \\
(74.4 \% / 18.2 \% / 6.5 \%)\end{array}$ & $\begin{array}{l}586 / 131 / 32 \\
(77.6 \% / 17.4 \% / 4.2 \%)\end{array}$ & $\begin{array}{l}278 / 67 / 16 \\
(77 \% / 18.6 \% / 4.4 \%)\end{array}$ \\
\hline Tumour size, $\mathrm{cm}$ & $6.1(3.9-9.6)$ & $3(2.4-3.9)$ & $6.5(5.2-8.1)$ & $12.1(11-14)$ \\
\hline $\begin{array}{l}\text { Tumour number } \\
\text { (solitary/multifocal) }\end{array}$ & $\begin{array}{l}895 / 654 \\
(57.8 \% / 42.2 \%)\end{array}$ & $\begin{array}{l}314 / 119 \\
(72.5 \% / 27.5 \%)\end{array}$ & $\begin{array}{l}387 / 368 \\
(51.3 \% / 48.7 \%)\end{array}$ & $\begin{array}{l}195 / 166 \\
(54 \% / 46 \%)\end{array}$ \\
\hline $\begin{array}{l}\text { AFP }(\leq 200 \mathrm{ng} \cdot \mathrm{ml}- \\
1 />200 \mathrm{ng} \cdot \mathrm{ml}-1)\end{array}$ & $\begin{array}{l}843 / 706 \\
(54.4 \% / 45.6 \%)\end{array}$ & $\begin{array}{l}281 / 152 \\
(64.9 \% / 35.1 \%)\end{array}$ & $\begin{array}{l}417 / 338 \\
(55.2 \% / 44.8 \%)\end{array}$ & $\begin{array}{l}147 / 214 \\
(40.7 \% / 59.3 \%)\end{array}$ \\
\hline WBC, 109/L & $5.22(3.95-6.65)$ & $4.68(3.47-6.03)$ & $5.2(3.97-6.62)$ & $5.96(4.80-7.32)$ \\
\hline PLT, 109/L & $132(86-186)$ & $97(62-144)$ & $131(88-181)$ & $179(129-229)$ \\
\hline ALT, IU/L & $38(25-58)$ & $33(23-48)$ & $38(26-56)$ & $46(30-70)$ \\
\hline AST, IU/L & $43(30-63)$ & $36(26-51)$ & $41(29-61)$ & $57(42-84)$ \\
\hline ALB, g/L & $39.3(36.0-43.1)$ & $39.4(36.1-43.1)$ & $39.5(36.1-43.2)$ & $38.7(35.4-43.0)$ \\
\hline TBIL, $\mu \mathrm{mol} / \mathrm{L}$ & $16.2(11.8-23.0)$ & $17(12-24.1)$ & $15.8(11.4-22.4)$ & $16.1(12-22.1)$ \\
\hline BUN, mg/dl & $5.3(4.3-6.3)$ & $5.3(4.3-6.3)$ & $5.4(4.4-6.4)$ & $5.1(4.2-6.0)$ \\
\hline $\mathrm{Cr}, \mu \mathrm{mol} / \mathrm{L}$ & $72.6(62.6-84.0)$ & $73(63-82.5)$ & $73(62.6-85)$ & $71(61-82)$ \\
\hline INR & $1.07(1.00-1.14)$ & $1.09(1.02-1.20)$ & $1.06(1.0-1.13)$ & $1.06(0.99-1.12)$ \\
\hline \multicolumn{5}{|c|}{$\begin{array}{l}\text { Abbreviations: HBV, hepatitis B virus; IQR, interquartile range; ECOG PS, Eastern Cooperative Oncology Group } \\
\text { performance status; AFP, alpha-fetoprotein; WBC, white blood cell; RBC, red blood cell; PLT, platelet; INR, } \\
\text { international normalized ratio; ALT, alanine aminotransferase; AST, aspartate aminotransferase; ALB, albumin; } \\
\text { TBIL, total bilirubin; BUN, blood urea nitrogen; }\end{array}$} \\
\hline
\end{tabular}

\section{ORR and clinical factors associated with lack of response after initial TACE}

Initial and best ORR were $64.6 \%$ and $70.1 \%$, respectively (Supplementary table 2). The kappa concordance value between initial and best response was 0.87. Mean time to initial and best response was 41.8 (standard error, SE, 14.5) and 48.1 (SE 30.5) days, respectively. Notably, 82 of 547 (15.0\%) initial non-responders achieved response after repeated TACE. Among patients whose status of response changed after repeated TACE (such as PR for initial response, $\mathrm{CR}$ for best response after sessions of TACE), mean time to initial response and best response was 42 (SE 
14.5) and 112 (SE 63.4) days. Multivariate logistic regression showed that only ECOG PS (adjusted odds ratio 1.53

[1.2-1.95], $P=0.001$ ) and tumor burden (adjusted odds ratio 3.08 [2.55-3.71], $P<0.001$ ) were predictors of non-CR (Supplementary table 3).

\section{Prognostic value of initial and best ORR in the whole cohort}

The median OS was 28.0 (95\% Cl 25.6-30.3) months in the entire cohort. Regarding type of response, median OS were 43.7 (38.8-50.1), 28.5 (26.1-32.9), 23.8 (21.1-27.2), and 8.2 (7.2-10.0) months for patients with initial CR, PR, SD and PD, respectively (log-rank $P<0.001$ in all comparisons except for $P=0.023$ between initial PR and SD,

Supplementary Fig. 3A); and 44.4 (39.9-50.1), 30.4 (27.3-34.4), 19.5 (17.3-22.7), and 8.2 (7.0-9.4) months for best CR, PR, SD, and PD, respectively (all with log-rank $P<0.001$, Supplementary Fig. 3B). Moreover, patients with initial CR and subsequent CR presented similar OS (median OS 43.7 [38.8-50.1] vs. 49.5 [38.1-60.8] months, log-rank $P=$ 0.468), whereas patients with subsequent PR had longer OS than persistent non-responders (median OS 30.0 [26.734.1 ] vs. 13.6 [11.4-15.8], log-rank $P<0.001$, Supplementary Fig. 3C). In time-dependent Cox regression analysis, both initial and best response were independent predictors of OS, with adjusted HR values of $1.59([1.36-1.87], P<0.001)$ and 2.44 ([2.09-2.85], $P<0.001)$, respectively. (Table 2). 
Table 2

Univariate and multivariate analysis by time-dependent Cox proportional regression in 1549 patients.

\begin{tabular}{|c|c|c|c|c|c|c|}
\hline \multirow[t]{2}{*}{ Risk factors } & \multicolumn{2}{|c|}{ Univariate analysis } & \multicolumn{2}{|c|}{$\begin{array}{l}\text { Multivariate analysis } \\
\text { (model } 1)\end{array}$} & \multicolumn{2}{|c|}{$\begin{array}{l}\text { Multivariate analysis } \\
\text { (model 2) }\end{array}$} \\
\hline & $\mathrm{HR}(95 \% \mathrm{Cl})$ & $\begin{array}{l}P \\
\text { value }\end{array}$ & $\begin{array}{l}\text { Adjusted HR } \\
(95 \% \mathrm{Cl})\end{array}$ & $\begin{array}{l}P \\
\text { value }\end{array}$ & $\begin{array}{l}\text { Adjusted HR } \\
\text { (95\% Cl) }\end{array}$ & $\begin{array}{l}\mathrm{P} \\
\text { value }\end{array}$ \\
\hline \multirow{3}{*}{$\begin{array}{l}\text { Gender (male/female) } \\
\text { Age, year old } \\
\text { Aetiology (HBV/others) }\end{array}$} & $\begin{array}{l}1.17(0.97- \\
1.40)\end{array}$ & 0.101 & - & - & - & - \\
\hline & \multirow{2}{*}{$\begin{array}{l}0.99(0.98- \\
0.99)\end{array}$} & 0.001 & $0.99(0.98-0.99)$ & 0.030 & $0.99(0.98-0.99)$ & 0.018 \\
\hline & & 0.293 & - & - & - & - \\
\hline \multirow{2}{*}{$\begin{array}{l}\text { ECOG PS score }(1 / 0) \\
\text { Tumour number }\end{array}$} & \multirow{2}{*}{$\begin{array}{l}0.89(0.71- \\
1.11)\end{array}$} & \multirow{2}{*}{$<.001$} & $1.32(1.14-1.53)$ & \multirow{2}{*}{0.001} & $1.34(1.16-1.56)$ & \multirow[t]{2}{*}{$\begin{array}{l}< \\
0.001\end{array}$} \\
\hline & & & $1.09(1.04-1.13)$ & & $1.05(1.01-1.09)$ & \\
\hline Tumour size, cm & $\begin{array}{l}1.59(1.38- \\
1.82)\end{array}$ & 0.001 & $1.04(1.02-1.06)$ & 0.001 & $1.04(1.02-1.06)$ & 0.011 \\
\hline $\begin{array}{l}\operatorname{AFP}(>200 / \leq 200 \\
\left.\mathrm{ng} \cdot \mathrm{ml}^{-1}\right)\end{array}$ & $\begin{array}{l}1.13(1.09- \\
1.17)\end{array}$ & $<.001$ & $1.39(1.12-1.60)$ & $\dot{0} 0.001$ & $1.37(1.18-1.58)$ & \multirow{2}{*}{$\begin{array}{l}0.001 \\
< \\
0.001\end{array}$} \\
\hline$A L B, g / L$ & $\begin{array}{l}1.09(1.08- \\
1.11)\end{array}$ & $<.001$ & $\begin{array}{l}0.98(0.97-1.00) \\
1.01(1.00-1.02)\end{array}$ & $<.001$ & $1.00(1.00-1.01)$ & \\
\hline TBIL, $\mu \mathrm{mol} / \mathrm{L}$ & \multirow{2}{*}{$\begin{array}{l}1.51(1.32- \\
1.73)\end{array}$} & \multirow{2}{*}{$<.001$} & $1.00(0.99-1.00)$ & 0.011 & $1.00(0.99-1.00)$ & \multirow{2}{*}{$\begin{array}{l}0.017 \\
0.239\end{array}$} \\
\hline AST, UI/L & & & - & 0.026 & - & \\
\hline ALT, UI/L & $\begin{array}{l}0.97(0.96- \\
0.99)\end{array}$ & $<.001$ & $0.98(0.93-1.03)$ & 0.980 & $0.98(0.93-1.02)$ & 0.710 \\
\hline BUN, mg/dl & $1.01(1.01-$ & & - & - & - & \\
\hline $\mathrm{Cr}, \mu \mathrm{mol} / \mathrm{L}$ & $1.02)$ & 0.001 & $1.00(1.00-1.01)$ & 0.400 & $1.00(1.00-1.01)$ & 0.330 \\
\hline $\mathrm{PLT}, 10^{9} / \mathrm{L}$ & $\begin{array}{l}1.00(1.00- \\
1.01)\end{array}$ & $\begin{array}{l}0.359 \\
0.006\end{array}$ & $1.05(1.01-1.08)$ & - & $1.04(1.01-1.08)$ & $\begin{array}{l}- \\
0.523\end{array}$ \\
\hline & & 0.444 & & & & 0.001 \\
\hline & & $\hat{0} .001$ & & & & 0.001 \\
\hline & & $\dot{0} 001$ & & & & $<.001$ \\
\hline & & 0.045 & & & & \\
\hline & & & & & & \\
\hline WBC, mol & $\begin{array}{l}1.00(0.99- \\
1.00)\end{array}$ & 0.001 & $2.23(1.30-3.82)$ & 0.717 & $2.36(1.40-4.00)$ & \\
\hline INR & & $<0.001$ & $1.59(1.36-1.87)$ & 0.007 & - & \\
\hline $\begin{array}{l}\text { Initial response (no } \\
\text { OR/OR) }\end{array}$ & $\begin{array}{l}0.94(0.90- \\
0.98)\end{array}$ & & - & 0.004 & $2.44(2.09-2.85)$ & \\
\hline $\begin{array}{l}\text { Best response (no OR } \\
\text { /OR) }\end{array}$ & $\begin{array}{l}1.00(0.99- \\
1.00)\end{array}$ & & & $\begin{array}{l}< \\
0.001\end{array}$ & & \\
\hline & $\begin{array}{l}1.01(1.00- \\
1.01)\end{array}$ & & & - & & \\
\hline
\end{tabular}




\begin{tabular}{|c|c|c|c|c|c|c|}
\hline \multirow[t]{2}{*}{ Risk factors } & \multicolumn{2}{|c|}{ Univariate analysis } & \multicolumn{2}{|c|}{$\begin{array}{l}\text { Multivariate analysis } \\
\text { (model 1) }\end{array}$} & \multicolumn{2}{|c|}{$\begin{array}{l}\text { Multivariate analysis } \\
\text { (model 2) }\end{array}$} \\
\hline & $\mathrm{HR}(95 \% \mathrm{Cl})$ & $\begin{array}{l}P \\
\text { value }\end{array}$ & $\begin{array}{l}\text { Adjusted HR } \\
(95 \% \mathrm{Cl})\end{array}$ & $\begin{array}{l}P \\
\text { value }\end{array}$ & $\begin{array}{l}\text { Adjusted HR } \\
(95 \% \mathrm{Cl})\end{array}$ & $\begin{array}{l}P \\
\text { value }\end{array}$ \\
\hline & \multicolumn{6}{|l|}{$\begin{array}{l}1.06(1.03- \\
1.09)\end{array}$} \\
\hline & \multicolumn{6}{|l|}{$\begin{array}{l}1.60(1.01- \\
2.54)^{(}\end{array}$} \\
\hline & \multicolumn{6}{|l|}{$\begin{array}{l}1.99(1.74- \\
2.28)\end{array}$} \\
\hline & \multicolumn{6}{|l|}{$\begin{array}{l}2.80(2.43- \\
3.22)\end{array}$} \\
\hline $\begin{array}{l}\text { Abbreviations } \\
\text { Oncology Gro } \\
\text { platelet; INR, } \\
\text { albumin; TBIL }\end{array}$ & $\begin{array}{l}\text { atio; Cl, confi } \\
\text { ce status; AF } \\
\text { ormalized ra } \\
\text { 7; BUN, blood }\end{array}$ & $\begin{array}{l}\text { einterv } \\
\text { oha-fetc } \\
\text { LLT, alar }\end{array}$ & $\begin{array}{l}\text {; HBV, hepatitis } \\
\text { rotein; WBC, wh } \\
\text { e aminotransf } \\
\text {; OR, objective }\end{array}$ & $\begin{array}{l}\text { s; ECOG } \\
\text { od cell; } \\
\text { AST, asp }\end{array}$ & $\begin{array}{l}\text { S, Eastern Coop } \\
\text { C, red blood ce } \\
\text { tate aminotran }\end{array}$ & ; ALB, \\
\hline
\end{tabular}

\section{Stratified analyses of prognostication of ORR}

As predictors of ORR, both tumor burden and ECOG PS score interacted with initial/best ORRs in predicting OS (Supplementary table 4). Consequently, the prognostic value of ORR was further tested in subgroups where tumor burden $\left(T_{S}+T_{N}\right)$ was stratified into low $(\leq 6)$, intermediate ( $>6$ but $\left.\leq 12\right)$, and high $(>12)$ according to previously proposed "six-and-twelve" score(18), and ECOG PS score was stratified into 0 and 1 point.

Initial ORRs in low, intermediate and high tumor burden strata were $84.1 \%, 68.7 \%$, and $33.0 \%$, respectively, and best ORRs were $86.6 \%, 72.1 \%$, and $45.7 \%$, respectively (Table 3, Supplementary tables 5-7). The relative differences between initial and best ORR ([best ORR - initial ORR] / initial ORR) were 3.0\%, 4.9\%, and 38.5\% in these three strata, respectively; and kappa concordance coefficient values between initial and best response among were $0.92,0.88$, and 0.78 , respectively.

Table 3

Complete response (CR) rate and objective response rate (ORR) in patients with different tumour characteristics

\begin{tabular}{|lllll|}
\hline & $\begin{array}{l}\text { Low } \\
\text { tumour burden }\end{array}$ & Intermediate tumour burden & High & P \\
& & tumour burden & \\
\hline Initial CR rate & $51.7 \%$ & $22.9 \%$ & $2.5 \%$ & $<0.001$ \\
Best CR rate & $55.7 \%$ & $29.1 \%$ & $5.8 \%$ & $<0.001$ \\
Initial ORR & $84.1 \%$ & $68.7 \%$ & $33.0 \%$ & $<0.001$ \\
Best ORR & $86.6 \%$ & $72.1 \%$ & $45.7 \%$ & $<0.001$ \\
\hline Abbreviations: CR, complete response; ORR, objective response rate. & \\
\hline
\end{tabular}

Initial ORRs in PS 0 and 1 strata were 69.5\%, and 54.6\%, respectively; while best ORRs were 74.7\%, and 60.2\%, respectively (Supplementary table 8-10). The relative differences between initial and best ORRs were 7.5\%, and $10.3 \%$, respectively; and kappa values for these two strata being 0.88 , and 0.87 respectively. 
In terms of stratified survival, initial responders had significantly better survival than initial non-responders in low (median OS 47.2 [41.2-59.6] vs. 25.4 [18.9-NA] months, log-rank $P<0.001$, Fig. 1A) and intermediate tumor burden strata (median OS 32.1 [29.0-37.9] vs. 18.3 [12.7-22.2] months, log-rank $P<0.001$, Fig. 1C), but not in high tumor burden strata (median OS 16.8 [15.4-22.5] vs. 14.9 [12.0-18.2] months, log-rank $P=0.537$, Fig. 1E). In contrast, best responders had consistently better survival than non-responders in all strata (median OS 47.2 [41.6-59.6] vs. 22.7 [16.8-33.0], 33.4 [29.5-38.5] vs. 14.6 [11.3-19.6], and 24.8 [20.9-32.5] vs. 11.1 [8.7-14.1] months for low, intermediate, and high tumor burden, respectively, all with log-rank $P<0.001$, Fig. 1B, D, and F). Regarding PS score strata, survival probabilities significantly differ between responders and non-responders regardless of PS score and timing of assessment (all with log-rank $P<0.001$, Supplementary Fig. 4). Such consistency between initial and best ORR across ECOG PS 0 and 1 reveals that PS was indiscriminate of timing of ORR assessment, further PS-related analyses were not performed.

Time-dependent Cox regression analysis showed that both initial and best response independently predictors OS in low (adjusted HR 2.55 [1.73-3.76] and 2.96 [1.97-4.46], respectively, both with $P<0.001$, Supplementary table 11) and intermediate tumor burden strata (adjusted HR 1.81 [1.48-2.22] and 2.13 [1.73-2.61], respectively, both with $P<$ 0.001 , Supplementary table 12). Nevertheless, in patients with high tumor burden, best response (adjusted HR 2.61 [1.99-3.42], $P<0.001$ ), but not initial response (adjusted HR 1.08 [0.84-1.40], $P=0.537$, (Supplementary table 13) was identified as a predictor of OS. The current findings are consistent in most of the subgroups with different ages, gender, liver function, ECOG PS, etiologies, and AFP levels. (Fig. 2A-C).

\section{ORR by MRECIST as a potential surrogate endpoint}

There exist strong correlations between raw survival probability deciles and odds of both initial $(R=0.82$ [0.39-0.96], $P=0.004)$ and best ORR $(R=0.93$ [0.66-0.99], $P<0.001)$ in unstratified population (Supplementary Fig. 5). In stratified analyses, initial response showed a significant association with OS in low and intermediate tumor burden strata $(R=$ 0.84 [0.1-0.98], $P=0.035$, and $\mathrm{R}=0.74$ [0.15-0.94], $P=0.023$, respectively, Fig. 5A, C, and E), but not in high tumor burden strata $(\mathrm{R}=0.22$ [-0.47-0.7], $P=0.54)$. In contrast, best response showed relatively consistent correlations in all strata $(\mathrm{R}=0.97$ [0.73-1.00], $P=0.002 ; \mathrm{R}=0.90$ [0.54-0.98], $P=0.002$; and $\mathrm{R}=0.70$ [0.07-0.93], $P=0.035$, for low, intermediate and high tumor burden, respectively, Fig. 5B, D, and F).

\section{Discussion}

The current study indicates that the surrogacy of initial and best ORR is associated with tumor burden:

low/intermediate tumor burden does not discriminate the prognostic value of initial or best ORR, whereas high tumor burden compromises the sensitivity of initial ORR, thus best ORR appear to be superior. The strengths and novelty of this study lie in (1) nationwide multicenter study with the largest sample size focusing on the prognostic value of ORR after TACE in unresectable HCC with a wide range of tumor burden; (2) in-depth research of the interaction between tumor burden and initial/best ORR for the first time; and (3) the first study utilizing individual patient data to comprehensively analyse the surrogacy of ORR with a long follow-up period.

The median OS of 28 months in the current cohort was comparable to that of 30 months reported by EASL guideline(1). However, compared to our previous results of 33 months, this slightly inferior prognosis of current population might be caused by the inclusion of over thirty percent of patients with ECOG PS 1 score(18), in order to investigate our hypothesis on the timing of ORR as a surrogate within a more generalized population, rather than the optimal candidates of TACE as was studied in our previous research. Since a criteria for stratification is needed in the current study to compare initial and best ORR in patients with different conditions, we endorsed the "six-and-twelve"

Page $11 / 21$ 
score as the basis of stratification due to its adequate performance in Chinese patient population compared to other models $(18,25,26)$. Moreover, although this study utilized the major conclusions of our previous "six-and-twelve" study as a basis in principal analyses, the current research serves a more stretched purpose of individualized determination of timing of response, and providing a new answer towards the controversial question of the superiority of initial or best response, rather than prognosis evaluation at pre-TACE baseline without information on radiological response.

Qualified surrogates of OS should be accurate and valid with sufficient sensitivity to capture all net survival benefits $(27,28)$. However, previously reported ORRs vary from 52-84\% (Supplementary table 14)(6). Apart from the heterogeneity in TACE technique and study population, the timing of ORR might also account for this discrepancy: in the current study, the finding that about every 1 out of 7 initial non-responders (15.0\%) may become responders after repeated TACE (Supplementary table 2, 82 of 547 initial non-responders achieved response after repeated TACE). This is particularly relevant given the similar outcomes of patients with initial and subsequent CR, and the significant difference between patients with subsequent and persistent non-responders. It can be inferred that best response is indispensable in addition to initial response if all net benefits are to be captured(3).

Nevertheless, since correlations between initial/best ORRs and survival were similar $(R=0.93, P<0.001$ vs. $R=0.82, P$ $=0.004$ ), indicating that overall analyses could not determine the optimal timing of response, and patient stratification is necessary. Upon identification between initial/best ORR and tumor burden, we performed stratified analyses in low $(\leq 6)$, intermediate ( $>6$ but $\leq 12)$, and high $(>12)$ tumor burden(18). Interestingly, the relative difference between initial and best response augmented considerably as tumor burden increases (3.0\%, $4.9 \%$, and 38.5\%, respectively for initial/best ORR) (Table 3). Correspondingly, correlation between best ORR and survival was similar as that of initial ORR in low and intermediate tumor burden strata ( $0.97 \mathrm{vs.} 0.84$ and $0.90 \mathrm{vs.} 0.74$ ), but became obviously better in high tumor burden stratum (0.70 vs. 0.22). This discrepancy may also explain why similar prognostic values of initial and best ORR was reported by a previous study: less than four nodules in $80 \%$ patients and the median tumor diameter of $3.1 \mathrm{~cm}$ indicated predominance of low/intermediate tumor burden. In contrast, 361 patients (23\%) in the current study had high tumor burden, enabling the identification of discrepancy between initial and best ORRs(14).

The most probable explanation is the "ceiling effect" and "threshold effect": for patients with low/intermediate tumor burden, initial TACE alone might reach the "ceiling" of effectiveness with adequate tumor necrosis, which is supported by the trend that previous studies reporting an initial ORR of $52 \%-100 \%$ had a median or mean tumor diameter of 2.7 to $6.2 \mathrm{~cm}$ (Supplementary table 15); yet for patients with high tumor burden, one session is hardly sufficient to step over the "threshold" with adequate necrosis, and a greater disparity between initial and best response rates can be expected after two or more sessions. This hypothesis is supported by a previous study where initial non-responders tended to have higher tumor burden and presented a subsequent response rate approaching $50 \%$ after second TACE(15). Similarly, in our previous study on TACE plus sorafenib where $44 \%$ patients had tumor diameter $>10 \mathrm{~cm}$, $57 \%$ patients had received two or more sessions at the optimal timing of response evaluation(9). Significantly, in those patients whose response status changed, the mean time to best response was 112 days, that means 1 or 2 sessions of procedures was conducted after initial treatment. If response status was not improved in this duration after repeated TACE, further alternatives may be considered.

Our findings suggest the need of a trade-off in the timeliness and sensitivity of ORR at different time point. For patients with $T_{S}+T_{N} \leq 12$, the prognostic values of initial and best ORRs were almost equivalent. Seeking subsequent OR in initial non-responders may be difficult, but would waste the early availability of initial evaluation, thus initial ORR should be preferred. For patients with $T_{S}+T_{N}>12$, only best ORR has qualified surrogacy, and the timeliness of initial ORR should not overweight its inaccuracy. In this case, best response should be adopted. 
Furthermore, it should be noted that radiological response per se is not only a surrogate endpoint, but also the basis of scheduling on-demand TACE. The current findings indicate that if initial OR could not be achieved with TACE alone despite a low/intermediate tumor burden, an early decision of resorting to additional therapies or other treatments is worthy of consideration, since repeated TACE is unlikely to bring further improvement of ORR, furthermore, survival does not differ between non-responders to TACE and untreated patients, thus repeated TACE may not be recommended in cases where OR cannot be achieved by prior TACE(29). However, for patients with high tumor burden, initial non-response of TACE does not necessarily negate this first-line non-curative treatment, since subsequent response still stands a chance with repeated TACE, and survival benefits can be possible. Nonetheless, the substantially improved ORR after subsequent TACE still remains suboptimal, and OS is still unfavorable(18), thus alternative therapies, such as targeted therapy, immunotherapy, TACE combined with systematic therapy or other modalities may be options worthy of consideration for those patients to improve outcomes(30).

Of note, preservation of liver function is as important as achieving a high OR, because the goal of treatment is to prolong OS, initial or repeated TACE might deteriorate liver function, especially in high tumor burden. this might be one of reasons of poor prognosis of those patients. However, in the current study focusing on ORR as surrogacy of OS in patients receiving TACE (1), heterogeneity in compensated liver function (restricted to Child-Pugh A5-B7) and PS (restricted to ECOG PS 0-1) was less significant compared to the wide range of tumor burden. These might be the reasons why liver function was not qualified predictive factors of OR in the current cohort (Supplementary table 3), and different performance status scores could not discriminate the surrogacy of initial and best OR in the current study (Supplementary Fig. 4). Moreover, assessing response by mRECIST criteria were mainly based on tumor burden $(6,23)$, and on-demand TACE is scheduled upon viable-tumors (which is very likely influenced by tumor burden) but not upon liver function or PS - as long as they are well-reserved for tolerance. This difference may lead to a result that OR and its timing is more dependent of tumor burden, but less of liver function or PS.

There are some limitations in our study. Firstly, selection bias was unavoidable due to its retrospective nature. However, the inclusion of consecutive cases from multiple centers with a large sample size may minimize this risk. Secondly, the tumor characteristics and etiologies of Chinese patients might differ from those in Europe and America; however, the subgroup analysis suggested that our findings were consistent in patients with etiologies other than HBV. Still, we admit that further investigations with large sample size of other etiologies in different regions to confirm our results are necessary. Thirdly, other unrevealed predictors that were not included or detected in our study, might be more significant and specific, such as chemotherapy agents, genetic or other biological variables. Thus, the generalization of our findings should be cautious. Finally, future trial-level data are needed to confirm these findings $(27,28)$.

\section{Conclusions}

In summary, optimal timing of ORR assessment should be tailored according to tumor burden. For patients with $\mathrm{T}_{\mathrm{S}}+$ $T_{N} \leq 12$, initial ORR is optimal for its timeliness upon similar sensitivity with best ORR. For the remaining patients with high tumor burden, the prognostic value of initial ORR is suboptimal and best ORR should be preferred. Further validations in other populations and trial-level studies are needed to validate these findings and guide future trial design and clinical practice.

\section{Abbreviations}

HCC, hepatocellular carcinoma; TACE, transarterial chemoembolization; OS, overall survival; mRECIST, modified Response Evaluation Criteria in Solid Tumors; OR. objective response; ORR, Objective response rate; CR, complete 
response; PR, partial response; $S D$, stable disease (SD); PD, progressive disease; AASLD, the American Association for the Study of Liver Disease; EASL, the European Association for the Study of Liver; ECOG PS, Eastern Cooperative Oncology Group performance status; CT, computed tomographyor; MR, magnetic resonance; Ts, tumor size; Tn, tumor number; HR hazard ratio; Cl, confidence interval; HBV, hepatitis B virus; HCV, hepatitis C virus; AFP, alpha-fetoprotein; $\mathrm{IQR}$, interquartile range; SE, standard error.

\section{Declarations}

\section{Authors' contributions:}

\#邓these authors share the first authorship equally

Dongdong Xia, Qiuhe Wang: study concept and design, acquisition of data, analysis and interpretation of data, drafting of the manuscript, critical revision of the manuscript for important intellectual content, statistical analysis, and administrative, technical, or material support.

Wei Bai, Enxin Wang, Zhexuan Wang: acquisition of data, analysis and interpretation of data, critical revision of the manuscript for important intellectual content, statistical analysis, and administrative, technical, or material support.

Wei Mu, Junhui Sun, Ming Huang, Guowen Yin, Hailiang Li, Hui Zhao, Chunqing Zhang, Jing Li, Jianbing Wu, Xiaoli Zhu, Shufa Yang, Xingnan Pan, Jiaping Li, Zixiang Li, Guohui Xu, Haibin Shi, Hui Zhang, Yuelin Zhang, Rong Ding, Hui Yu, Lin Zheng, Xiaohu Yang, Guangchuan Wang, Nan You, Long Feng, Shuai Zhang, Wukui Huang, Tao Xu, Wenzhe Fan, Xueda Li, Xuegang Yang, Weizhong Zhou, Wenjun Wang, Qiaoyi Yang, Xiaomei Li, Bohan Luo, Jing Niu, Jie Yuan, Yong Lv, Jing Li, Jiahao Fan, Kai Li, Wengang Guo\&Zhanxin Yin, Daiming Fan: acquisition of data, critical revision of the manuscript for important intellectual content, and administrative, technical, or material support.

Jielai Xia: analysis and interpretation of data, critical revision of the manuscript for important intellectual content, statistical analysis.

Guohong Han: study concept and design, acquisition of data, analysis and interpretation of data, drafting of the manuscript, critical revision of the manuscript for important intellectual content, statistical analysis, and administrative, technical, or material support, and funding acquisition.

All authors approved the final version of the manuscript

\section{Ethics approval[}

Permission for retrospective data analyses was obtained from the Ethics Committees of all participating centers. The study was performed according to the ethical guidelines of the 1975 Declaration of Helsinki.

Consent to participate[Written informed consent was obtained from the patients.

Conflicts of interest: Nothing to declare for all authors

\section{Funding Sources:}

This study was supported by National Natural Science Foundation of China (81172145 and 81420108020$)$ and National Key Technology R\&D Program (2015BAl13B07). 


\section{Code availability冈NA}

\section{References}

1. European Association for the Study of the Liver. Electronic address eee, European Association for the Study of the L. EASL Clinical Practice Guidelines: Management of hepatocellular carcinoma. J Hepatol 2018;69:182-236.

2. Heimbach JK, Kulik LM, Finn RS, Sirlin CB, Abecassis MM, Roberts LR, Zhu AX, et al. AASLD guidelines for the treatment of hepatocellular carcinoma. Hepatology 2018;67:358-380.

3. Llovet JM, Montal R, Villanueva A. Randomized trials and endpoints in advanced HCC: Role of PFS as a surrogate of survival. J Hepatol 2019;70:1262-1277.

4. Riaz A, Miller FH, Kulik LM, Nikolaidis P, Yaghmai V, Lewandowski RJ, Mulcahy MF, et al. Imaging response in the primary index lesion and clinical outcomes following transarterial locoregional therapy for hepatocellular carcinoma. JAMA 2010;303:1062-1069.

5. Han G, Berhane S, Toyoda H, Bettinger D, Elshaarawy O, Chan AWH, Kirstein M, et al. Prediction of Survival Among Patients Receiving Transarterial Chemoembolization for Hepatocellular Carcinoma: A Response-Based Approach. Hepatology 2020;72:198-212.

6. Llovet JM, Lencioni R. mRECIST for HCC: Performance and novel refinements. J Hepatol 2020;72:288-306.

7. Gillmore R, Stuart S, Kirkwood A, Hameeduddin A, Woodward N, Burroughs AK, Meyer T. EASL and mRECIST responses are independent prognostic factors for survival in hepatocellular cancer patients treated with transarterial embolization. J Hepatol 2011;55:1309-1316.

8. Prajapati HJ, Spivey JR, Hanish SI, El-Rayes BF, Kauh JS, Chen Z, Kim HS. mRECIST and EASL responses at early time point by contrast-enhanced dynamic MRI predict survival in patients with unresectable hepatocellular carcinoma (HCC) treated by doxorubicin drug-eluting beads transarterial chemoembolization (DEB TACE). Ann Oncol 2013;24:965-973.

9. Liu L, Wang W, Chen H, Zhao Y, Bai W, Yin Z, He C, et al. EASL- and mRECIST-evaluated responses to combination therapy of sorafenib with transarterial chemoembolization predict survival in patients with hepatocellular carcinoma. Clin Cancer Res 2014;20:1623-1631.

10. Memon K, Kulik L, Lewandowski RJ, Wang E, Riaz A, Ryu RK, Sato KT, et al. Radiographic response to locoregional therapy in hepatocellular carcinoma predicts patient survival times. Gastroenterology 2011;141:526-535, 535 e521-522.

11. Wang W, Zhao Y, Bai W, Han G. Response assessment for HCC patients treated with repeated TACE: The optimal time-point is still an open issue. J Hepatol 2015;63:1530-1531.

12. Riaz A, Memon K, Miller FH, Nikolaidis P, Kulik LM, Lewandowski RJ, Ryu RK, et al. Role of the EASL, RECIST, and WHO response guidelines alone or in combination for hepatocellular carcinoma: radiologic-pathologic correlation. J Hepatol 2011;54:695-704.

13. Huo TI, Huang YH, Wu JC, Lee PC, Chang FY, Lee SD. Induction of complete tumor necrosis may reduce intrahepatic metastasis and prolong survival in patients with hepatocellular carcinoma undergoing locoregional 
therapy: a prospective study. Ann Oncol 2004;15:775-780.

14. Kim BK, Kim SU, Kim KA, Chung YE, Kim MJ, Park MS, Park JY, et al. Complete response at first chemoembolization is still the most robust predictor for favorable outcome in hepatocellular carcinoma. J Hepatol 2015;62:1304-1310.

15. Georgiades C, Geschwind JF, Harrison N, Hines-Peralta A, Liapi E, Hong K, Wu Z, et al. Lack of response after initial chemoembolization for hepatocellular carcinoma: does it predict failure of subsequent treatment? Radiology 2012;265:115-123.

16. Shim JH, Lee HC, Kim SO, Shin YM, Kim KM, Lim YS, Suh DJ. Which response criteria best help predict survival of patients with hepatocellular carcinoma following chemoembolization? A validation study of old and new models. Radiology 2012;262:708-718.

17. Kim DY, Ryu HJ, Choi JY, Park JY, Lee DY, Kim BK, Kim SU, et al. Radiological response predicts survival following transarterial chemoembolisation in patients with unresectable hepatocellular carcinoma. Aliment Pharmacol Ther 2012;35:1343-1350.

18. Wang Q, Xia D, Bai W, Wang E, Sun J, Huang M, Mu W, et al. Development of a prognostic score for recommended TACE candidates with hepatocellular carcinoma: A multicentre observational study. J Hepatol 2019;70:893-903.

19. Kudo M, Izumi N, Kokudo N, Matsui O, Sakamoto M, Nakashima O, Kojiro M, et al. Management of hepatocellular carcinoma in Japan: Consensus-Based Clinical Practice Guidelines proposed by the Japan Society of Hepatology (JSH) 2010 updated version. Dig Dis 2011;29:339-364.

20. Cheng AL, Amarapurkar D, Chao Y, Chen PJ, Geschwind JF, Goh KL, Han KH, et al. Re-evaluating transarterial chemoembolization for the treatment of hepatocellular carcinoma: Consensus recommendations and review by an International Expert Panel. Liver Int 2014;34:174-183.

21. Kim BK, Kim KA, Park JY, Ahn SH, Chon CY, Han KH, Kim SU, et al. Prospective comparison of prognostic values of modified Response Evaluation Criteria in Solid Tumours with European Association for the Study of the Liver criteria in hepatocellular carcinoma following chemoembolisation. Eur J Cancer 2013;49:826-834.

22. Sieghart W, Hucke F, Pinter M, Graziadei I, Vogel W, Muller C, Heinzl H, et al. The ART of decision making: retreatment with transarterial chemoembolization in patients with hepatocellular carcinoma. Hepatology 2013;57:2261-2273.

23. Lencioni R, Llovet JM. Modified RECIST (mRECIST) assessment for hepatocellular carcinoma. Semin Liver Dis 2010;30:52-60.

24. Lencioni R, Montal R, Torres F, Park JW, Decaens T, Raoul JL, Kudo M, et al. Objective response by mRECIST as a predictor and potential surrogate end-point of overall survival in advanced HCC. J Hepatol 2017;66:1166-1172.

25. Mazzaferro V, Llovet JM, Miceli R, Bhoori S, Schiavo M, Mariani L, Camerini T, et al. Predicting survival after liver transplantation in patients with hepatocellular carcinoma beyond the Milan criteria: a retrospective, exploratory analysis. Lancet Oncol 2009;10:35-43. 
26. Yamakado K, Miyayama S, Hirota S, Mizunuma K, Nakamura K, Inaba Y, Maeda H, et al. Subgrouping of intermediate-stage (BCLC stage B) hepatocellular carcinoma based on tumor number and size and Child-Pugh grade correlated with prognosis after transarterial chemoembolization. Jpn J Radiol 2014;32:260-265.

27. Zhao F. Surrogate End Points and Their Validation in Oncology Clinical Trials. J Clin Oncol 2016;34:1436-1437.

28. Buyse $M$, Molenberghs $\mathrm{G}$. Criteria for the validation of surrogate endpoints in randomized experiments. Biometrics 1998;54:1014-1029.

29. Llovet JM, Real MI, Montana X, Planas R, Coll S, Aponte J, Ayuso C, et al. Arterial embolisation or chemoembolisation versus symptomatic treatment in patients with unresectable hepatocellular carcinoma: a randomised controlled trial. Lancet 2002;359:1734-1739.

30. Palmer DH, Malagari K, Kulik LM. Role of locoregional therapies in the wake of systemic therapy. J Hepatol 2020;72:277-287.

\section{Figures}



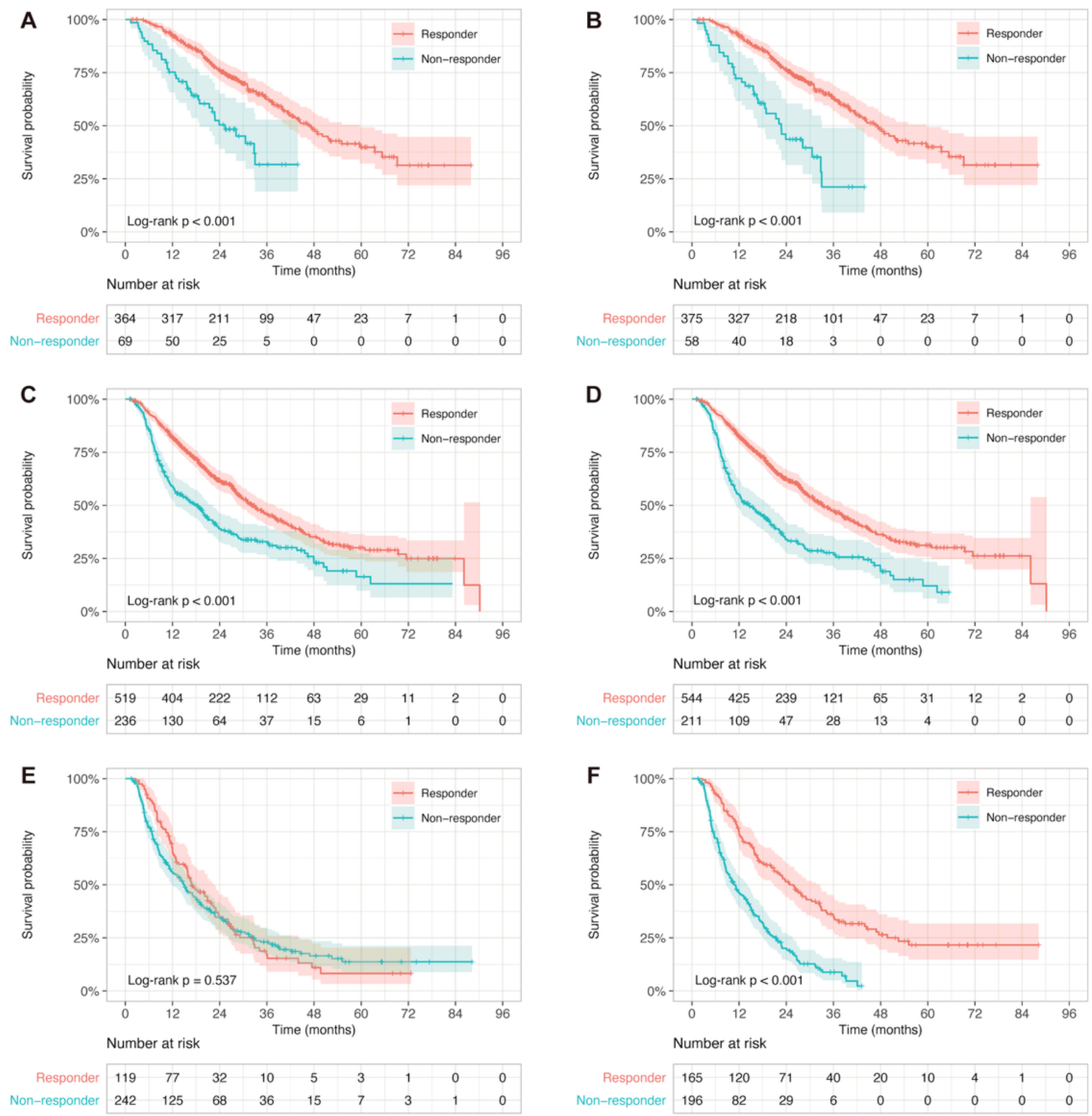

Figure 1

Survival curves of responders and non-responders identified by initial and best response stratified by tumor burden. (A) Survival of initial responders vs. non-responders within low tumor burden stratum. Median OS were 47.2 (41.2$59.6)$ vs. 25.4 (18.9-NA) months, log-rank p<0.001. (B) Survival of best responders vs. non-responders within low tumor burden stratum. Median OS were 47.2 (41.6-59.6) vs. 22.7 (16.8-33.0) months, log-rank $p<0.001$. (C) Survival of initial responders vs. non-responders within intermediate tumor burden stratum. Median OS were 32.1 (29.0-37.9) vs. 18.3 (12.7-22.2) months, log-rank $P<0.001$. (D) Survival of best responders vs. non-responders within intermediate tumor burden stratum. Median OS were 33.4 (29.5-38.5) vs. $14.6(11.3-19.6)$ months, log-rank $p<0.001$. (E) Survival of initial responders vs. non-responders within high tumor burden stratum. Median OS were 16.8 (15.4-22.5) vs. 14.9 (12.0-18.2) months, log-rank $P=0.537$. (F) Survival of best responders vs. non-responders within high tumor burden stratum. Median OS were and 24.8 (20.9-32.5) vs. 11.1 (8.7-14.1) months, log-rank $\mathrm{P}<0.001$. 


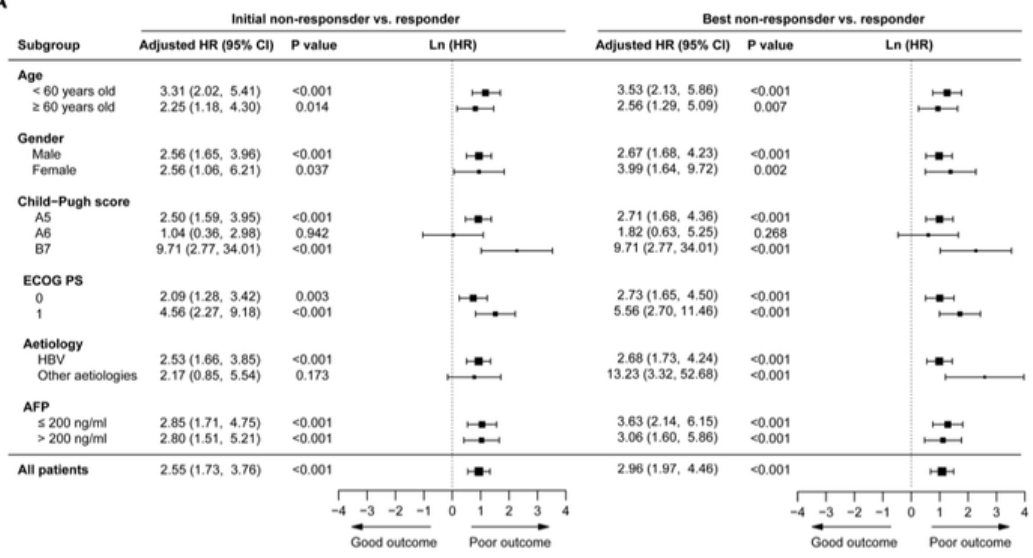

B

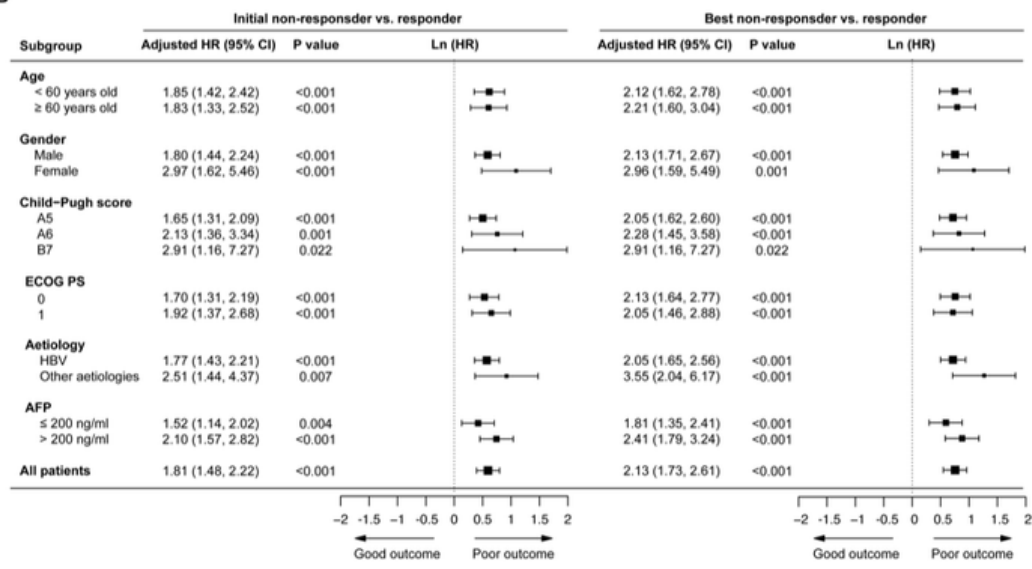

C

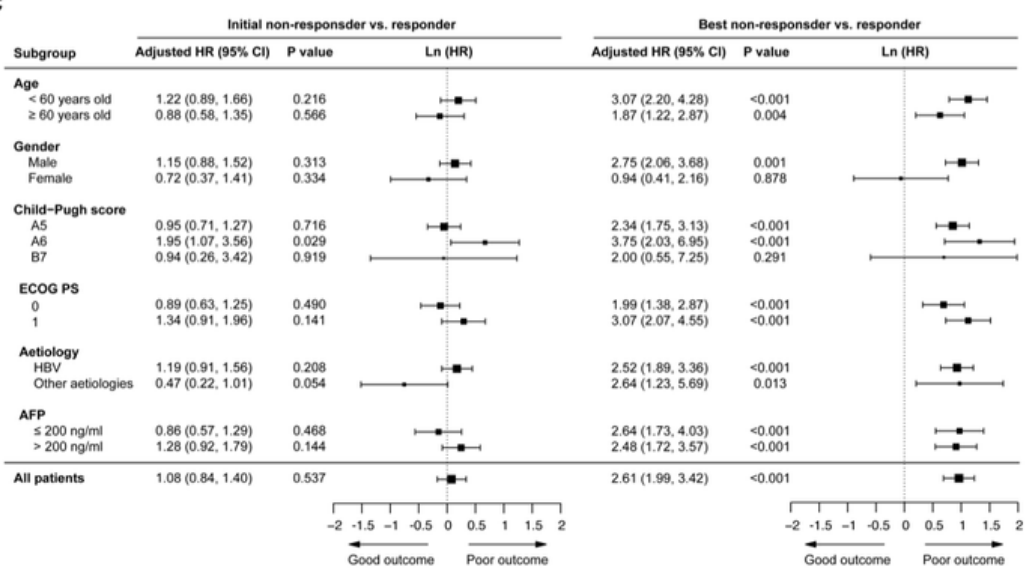

\section{Figure 2}

Forest plot of HR (multiplicative scale) for initial and best response in predicting OS stratified by tumour burden. (A) HR for initial and best response in predicting OS within low tumour burden strata. Both initial and best response presented robust prognostic value across most subgroups. (B) HR for initial and best response in predicting OS within intermediate tumour burden strata. Both initial and best response presented robust prognostic value across most subgroups. (C) HR for initial and best response in predicting OS within high tumour burden strata. Initial response was not predictive of OS in any subgroup, whereas best response remained robust in most subgroups. 
A

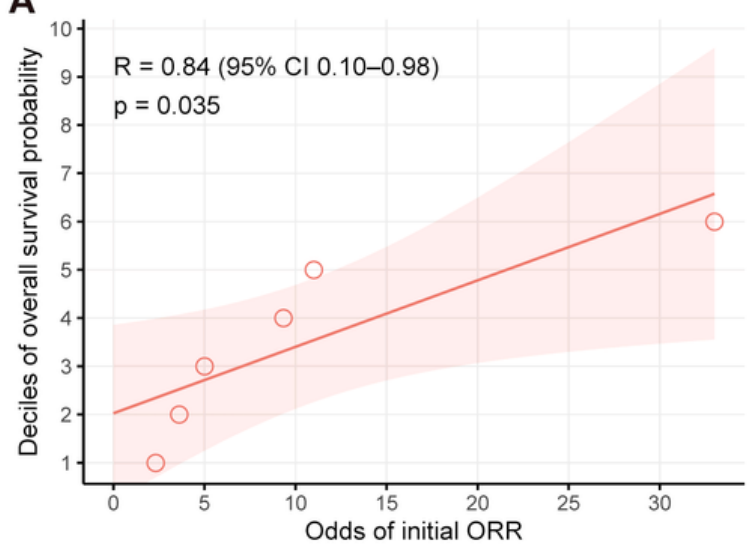

C
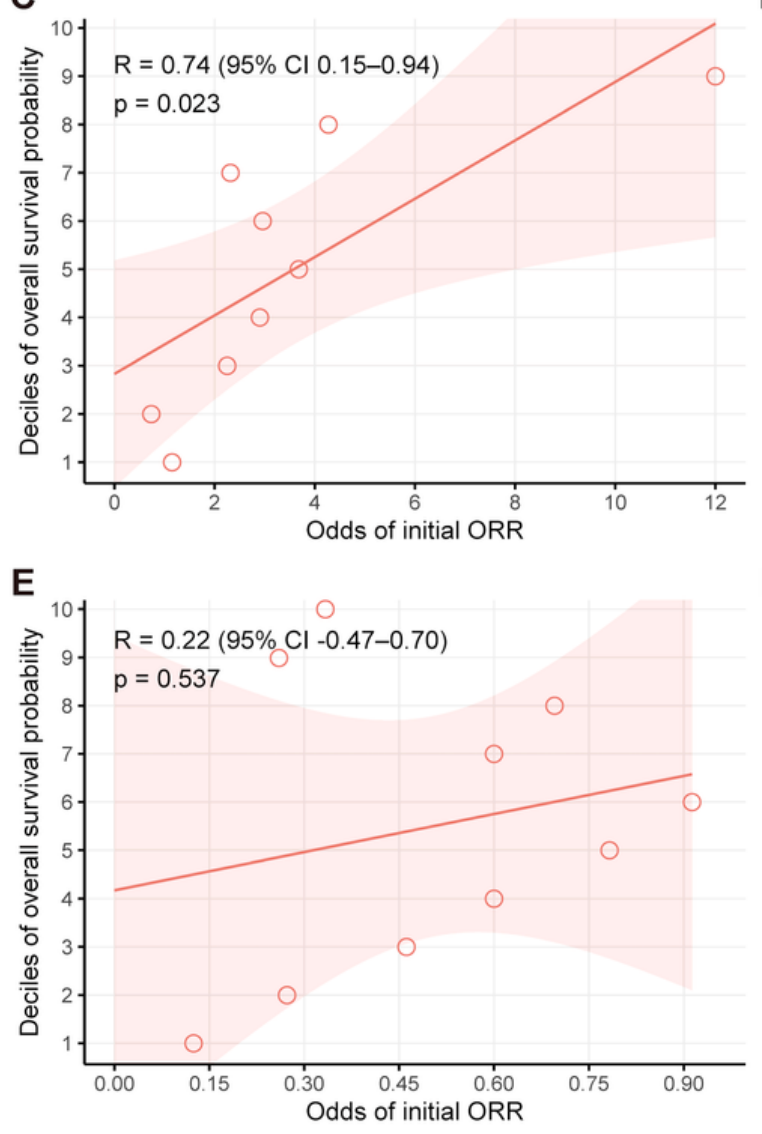

B

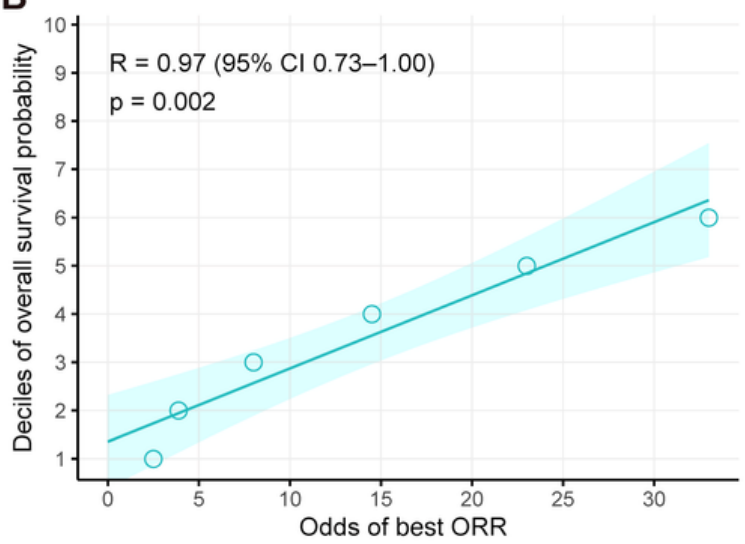

D

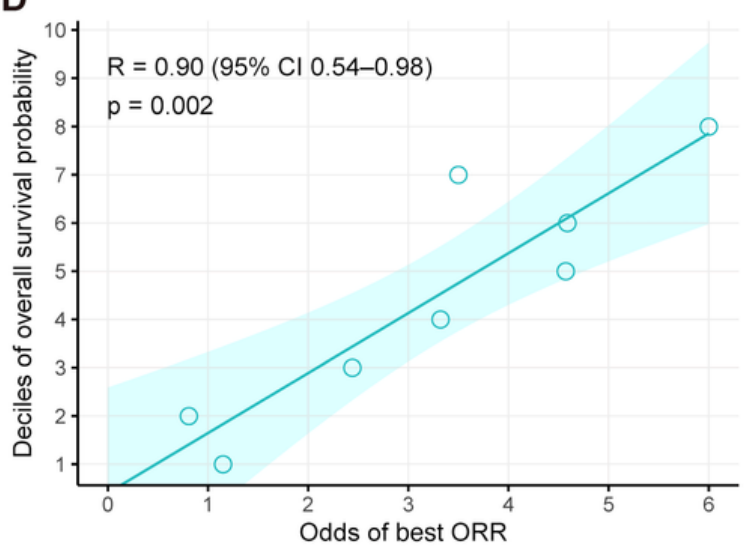

$\mathbf{F}$

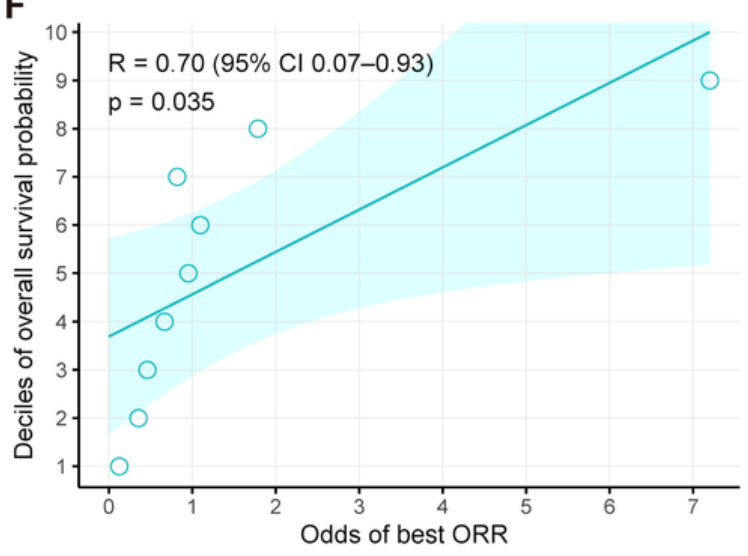

\section{Figure 3}

Correlation between odds of ORR and survival probability in deciles stratified by tumor burden. (A) Odds of initial ORR was strongly correlated with survival probability in deciles within low tumor burden stratum, $\mathrm{R}=0.84(95 \% \mathrm{Cl} 0.10-$ 0.98), $p=0.035$. (B) Odds of best ORR was strongly correlated with survival probability in deciles within low tumor burden stratum, $R=0.97(95 \% \mathrm{Cl} 0.73-1.00), p=0.002$. (C) Odds of initial ORR was correlated with survival probability in deciles within intermediate tumor burden stratum, $R=0.74(95 \% \mathrm{Cl} 0.15-0.94), p=0.023$. (D) Odds of best ORR was strongly correlated with survival probability in deciles within intermediate tumor burden stratum, $\mathrm{R}=0.90(95 \% \mathrm{Cl}$ 0.54-0.98), $p=0.002$. (E) No significant correlation between odds of initial ORR and survival probability in deciles was observed in high tumor burden stratum, $R=0.22(95 \% \mathrm{Cl}-0.47-0.70), p=0.537$. (F) Odds of best ORR was correlated with survival probability in deciles within high tumor burden stratum, $R=0.70(95 \% \mathrm{Cl} 0.07-0.93), p=0.035$. 


\section{Supplementary Files}

This is a list of supplementary files associated with this preprint. Click to download.

- Supplementarymaterials.docx 